\title{
Neurotrophic Effects of Vascular Endothelial Growth Factor on Organotypic Cortical Explants and Primary Cortical Neurons
}

\author{
Jeffrey M. Rosenstein, Nina Mani, Alfia Khaibullina, and Janette M. Krum \\ Department of Anatomy and Cell Biology, The George Washington University Medical Center, Washington, DC 20037
}

\begin{abstract}
Vascular endothelial growth factor (VEGF) is well known to play an important regulatory role in vascular growth and development. Because gene knock-outs of VEGF and its receptors $f l k-1$ and $f l t-1$ result in early embryonic lethality, determining roles for VEGF in CNS development has been particularly difficult. Recent studies have shown that VEGF is upregulated after various injuries to the adult brain and that the cytokine affords protection to cultured neurons affected by oxidative or excitotoxic stress. The present study demonstrates, for the first time, that VEGF is directly neurotrophic to CNS neurons in culture. We applied VEGF to normoxic fetal organotypic cortical explants as a model of CNS neuropil, in addition to primary cortical neurons, to assess direct growth effects absent vascular or astroglial activity. We found that VEGF provided a significant dose-responsive increase in the neuronal microtubule markers TUJ1 and MAP-2, as well as mRNA for MAP-2 and $f l k-1$. Antisense oligodeoxynucleotides to $f l k-1$, but not $f l t-1$, inhibited neuritic outgrowth, whereas inhibitors of the signaling pathways MEK1 and P13-AKT both abrogated VEGF-induced growth. VEGF applied to primary cortical neurons produced significant increases in neuronal cell body diameter and the number of emerging neurites mediated by flk-1. Possibly, VEGF achieves its effects by acting on the neuronal microtubular content, which is involved with growth, stability and maturation. Several studies have now shown that VEGF is neurotrophic and neuroprotective independent of a vascular component; we suggest that VEGF plays seminal pleiotrophic roles in CNS development and repair.
\end{abstract}

Key words: VEGF; neurotrophic factor; explant culture; cortex; receptor; signal transduction

\section{Introduction}

Vascular endothelial growth factor (VEGF) is a secreted vascular mitogen that is purportedly specific for endothelial cells and plays an important regulatory role in vascular growth in several organ systems during development (Ferrara and Davis-Smyth, 1997). VEGF has been implicated as the predominant angiogenic factor in tumors, epithelial wound healing, and diabetic retinopathy (Dvorak et al., 1995). Little information exists concerning the direct growth effects of the application of VEGF to CNS tissue because most work regarding VEGF action, receptor mechanisms, and signaling pathways has been done in non-neural tissues. The importance of VEGF and its primary receptors flk-1 (KDR/VEGFR2) and flt-1 (VEGFR1) is illustrated by knock-outs of their genes, which results in severe vascular defects and early embryonic death (Fong et al., 1995; Shalaby et al., 1995; Carmeliet et al., 1996). Thus, precise growth and developmental roles for VEGF in CNS tissue are difficult to determine, particularly because the protein is markedly downregulated after birth. However, VEGF and its receptors are strongly upregulated in several

\footnotetext{
Received June 18, 2003; revised 0ct. 8, 2003; accepted 0ct. 13, 2003.

This work was supported by National Institutes of Health (NIH) Grant NS-39282 (J.M.R.) and in part by NIH Grant NS-38128 (J.M.K.). We thank Newton More and Gauri Tadvalkar for technical assistance.

Correspondence should be addressed to Dr. Jeffrey M. Rosenstein, Department of Anatomy and Cell Biology, The George Washington University Medical Center, 2300 I Street Northwest, Washington, DC 20037. E-mail: anajmr@gwumc.edu.

Copyright $\odot 2003$ Society for Neuroscience 0270-6474/03/2311036-09\$15.00/0
}

cell types after stroke or mechanical injury (Kovacs et al., 1996; Krum and Rosenstein, 1998; Marti and Risau, 1999).

Recent studies have indicated that VEGF may have additional actions beyond vascularization that point to a neurotrophic role in nervous tissue. When VEGF was applied to fetal ventral mesencephalic explants, a substantial increase in neuritic outgrowth was found as well as enhanced survival of dopaminergic neurons compared with nontreated explants (Silverman et al., 1999). Further, VEGF application to cultured peripheral adult ganglia caused significant neuritic outgrowth that was mediated by the flk-1 receptor (Sondell et al., 1999). Peripheral nerve regeneration in vivo has been shown to be enhanced by VEGF application, possibly because of increased angiogenesis and Schwann cell populations (Hobson et al., 2000; Schratzberger et al., 2000). VEGF has also been implicated in a neuroprotective role in vitro by rescuing both primary cortical neurons after ischemia or serum deprivation (Jin et al., 2000a,b) and primary hippocampal neurons after excitotoxicity (Matsuzaki et al., 2001; Svensson et al., 2002).

We have developed a serum-free organotypic explant system using embryonic day 17 (E17) to E18 rat brain to understand how exogenous VEGF application may affect CNS neuropil development (Rosenstein et al., 1998; Silverman et al., 1999). In the present study we have examined the neurotrophic actions of VEGF on fetal cortical explants as well as on primary cortical neurons to further assess how VEGF may directly affect neuronal 
growth absent any angiogenic or astroglial activity. The results show that VEGF application to explants produces substantial and specific flk-1 receptor-mediated neuritic growth and maturation that is likely dependent on both the phosphatidylinositol 3-kinase (PI3-K)/Akt (serine/threonine protein kinase) and the mitogen-activated protein kinase kinase (MEK/ERK) signaling pathways. In primary neuronal cultures, VEGF caused significant neuritic outgrowth and increased somal size compared with untreated neurons. By upregulating developmentally regulated neuronal proteins related to microtubule function or metabolism, we suggest that VEGF plays an important role in the growth, development, and potentially, structural stability of CNS neurons.

\section{Materials and Methods}

Neocortical explant preparation and incubation. Pregnant Wistar dams (E17-E18) were anesthetized with ketamine $(60 \mathrm{mg} / \mathrm{kg}) / x y l a z i n e$ (Rompun; $6 \mathrm{mg} / \mathrm{kg}$ ), and the fetal brains were removed under aseptic conditions. The meningeal coverings were removed and the neocortex was dissected in ice-cold Earle's balanced salt solution (Invitrogen, Grand Island, NY). A Stoelting (Kiel, WI) manual tissue slicer was used to produce $400 \mu \mathrm{m}$ cortical slices. Six slices of fetal tissue were arranged on Millicell-CM culture plate inserts (Millipore, Bedford, MA), and the inserts were placed in six-well culture dishes. Each well received $1 \mathrm{ml}$ of serum-free medium consisting of Neurobasal medium (modified DMEM-F12) with B27 supplement, $25 \mu \mathrm{M}$ glutamate and $0.5 \mathrm{~mm}$ glutamine (Invitrogen) added. Experimental cultures received doses of human recombinant $\operatorname{VEGF}_{165}$ (R \& D Systems, Minneapolis, MN) of 1, 10, 25,50 , or $100 \mathrm{ng} / \mathrm{ml}$ added directly to the medium immediately before introduction of the slices; control explants (the sixth well) did not receive VEGF $_{165}$. All explant cultures were incubated identically under standard conditions for $3 \mathrm{~d}$. Separate sets of explants (fresh frozen) were used for reverse transcriptase (RT)-PCR analysis.

Primary cortical neuronal cultures. Cerebral cortical neurons were isolated from E18 rat fetuses and cultured in serum-free medium. Fetuses were delivered by cesarean section and decapitated in ice; the meninges were removed from the brain and the neocortex was dissected out. The combined neocortices from three to four fetuses were cut into $1 \mathrm{~mm}$ cubes, briefly trypsinized and triturated $10-15$ times through a firepolished Pasteur pipette in a tube containing $2 \mathrm{ml}$ of B27/neurobasal medium. After any undispersed tissue settled, the supernatant was spun down for $1 \mathrm{~min}$ at $200 \times \mathrm{g}$. The number of viable cells was determined with a hemocytometer, and the desired neuronal density ( $\sim 200$ cells/ $\mathrm{mm}^{2}$ ) was obtained by dilution of the primary cell suspension in Neurobasal medium supplemented with $0.5 \mathrm{~mm}$ glutamine, $25 \mu \mathrm{M}$ glutamate, and B27 supplement (Invitrogen) with supplemented neuronal medium.

The cultures were plated on sterile poly-D-lysine/laminin-coated glass coverslips in six-well culture dishes in fresh B27/Neurobasal medium containing the various dosages of VEGF [ 0 (control), 1, 10, 25, 50, or 100 $\mathrm{ng} / \mathrm{ml}$. The cultures were then incubated for $3 \mathrm{~d}$ under standard conditions. Immunohistochemical analysis of GFAP expression was used to confirm the purity of neuronal cultures. In the majority of experiments, the cultures consisted of $\sim 95 \%$ neurons.

Immunocytochemistry. Immunocytochemical evaluation of explants was conducted after overnight fixation of whole explants $(2 \mathrm{hr}$ for primary neuronal cultures) at $4^{\circ} \mathrm{C}$ in a fixative solution of $4 \%$ paraformaldehyde, $0.1 \mathrm{~m}$ sodium cacodylate buffer, and $3 \%$ sucrose, $\mathrm{pH}$ 7.2. After washing in $0.05 \mathrm{M}$ Tris-buffered saline (TBS), $\mathrm{pH} 7.6$, the cultures were processed for immunochemistry. Endogenous peroxidase activity was blocked by incubating explants for $10 \mathrm{~min}$ in $3 \%$ hydrogen peroxide in $10 \%$ methanol, at room temperature. This was followed by incubation in the appropriate primary antisera dilutions for $48 \mathrm{hr}$ at $4^{\circ} \mathrm{C}$. After washing with $0.05 \mathrm{~m}$ TBS, the explants were incubated with the appropriate secondary antibody for $30 \mathrm{~min}$, followed by incubation for $30 \mathrm{~min}$ with either rabbit or mouse PAP (Sigma, St. Louis, MO), and reaction products were visualized by diaminobenzidine. Other cultures were subjected to confocal analysis after single or double immunofluorescence staining. In these specimens, FITC- or Texas Red-conjugated secondary antibodies were used (Vector Laboratories, Burlingame, CA). All tissues were scanned using Lasersharp software (Bio-Rad, Hercules, CA) at 20× magnification using a Bio-Rad 1024 laser confocal microscope at 10-30\% laser excitation at a Z-series optical sectioning thickness of $0.5-1 \mu \mathrm{m}$. Images were collected in sequential mode, using an image scan filtering Kalman range of 4-6, reassembled as projections using Lasersharp software, and then merged using Bio-Rad plug-ins in Photoshop (Adobe Systems, San Jose, CA). Immunocytochemical analysis of various antigens was repeated at least three times for each antigen and experimental procedure.

Controls included omission of primary antibodies and replacement of the primary antibody with normal serum. The antibodies used were microtubule-associated protein (MAP-2) (1:500; Boehringer Mannheim, Ridgefield, CT), neuronal nuclear antigen (NeuN) (1:500; Chemicon, Temecula, CA), neuron specific enolase (NSE; 1:500; Polysciences, Warrington, PA), and $\beta$-tubulin III (TUJ1; 1:500; Covance, Berkeley, CA); flt-1 (1:50; Santa Cruz Biotechnology, Santa Cruz, CA) and flk-1 (1:50; Santa Cruz Biotechnology) or 1:100 (Calbiochem, La Jolla, CA) were used to identify VEGF receptor markers.

Quantification of immunostained cultures. Images of PAP MAP-2 immunostaining for each VEGF dose and control were digitized using the Image-Pro image analysis system (Media Cybernetics, Silver Spring, $\mathrm{MD}$ ) with Metamorph software (Universal Imaging, Downingtown, PA) images of 6.4 inches $\times 4.8$ inches captured at $20 \times$ magnification. To measure the relative area occupied by neurons and to exclude variations in $\mathrm{DAB}$ immunostaining intensity, the threshold color value below which staining would not be counted was determined and the area above the threshold measured as a ratio to the total area analyzed. For each VEGF dose and control explants, no fewer than three fetal brains were used for semiquantitative analysis, and six random regions within each were sampled. The data represents the results from four experiments. Statistical significance was evaluated using Student's $t$ and Mann-Whitney tests (Sigma Stat 2.03; SPSS Inc., Chicago, IL). The difference between control and VEGF-treated samples was considered to be statistically significant at $p \leq 0.001$.

For measurements in primary cortical neuronal cultures, bright-field images immunostained with TUJ1 antibody were obtained and digitized with a Coolsnap Pro (Nikon, Media Cybernetics) color camera. All images for each series were acquired using ImagePro Express 3.2 (Media Cybernetics) during one session, with the same brightness settings. Before measurements, ImagePro Express was calibrated using a commercial $1 \mathrm{~mm}$ calibration slide for $20 \times$ or $40 \times$ magnification microscope setting. Therefore, the neuronal soma diameter was acquired in absolute units (micrometers) and the data were exported into Excel (Microsoft, Seattle, WA) workbook. From each VEGF dose in a series, three to four random areas from each coverslip slide were collected for measurements. Neurons with an entire nuclear profile were measured across their broadest diameter. Neurites were counted only if they had an obvious attachment to the neuronal soma, and more distal branching patterns were not measured because neuritic outgrowth was so robust after VEGF application. For the statistical analysis, one-way ANOVA and the Mann-Whitney rank sum test were performed (Sigma Stat 2.03; SPSS Inc.). The difference between control and VEGF-treated samples was considered to be statistically significant at $p \leq 0.001$.

Semiquantitative RT-PCR. Fresh-frozen tissue from explants was used to obtain total RNA using the Purescript RNA isolation kit (Gentra Systems, Minneapolis, MN). RNA from each sample was reversetranscribed using the Superscript preamplification System for First Strand cDNA Synthesis kit (Invitrogen). Normalization of cDNA samples was performed using the internal standard glyceraldehyde-3phosphate dehydrogenase (G3PDH). Amplification of each of the genes being tested was performed at the exponential phase (number of cycles determined for each tested gene) using the normalized template values. PCR products were resolved by $1.2 \%$ agarose gel electrophoresis and stained with ethidium bromide and visualized under UV light. After this, direct comparisons of the relative abundance of gene-specific mRNAs were made. In general, the PCR mixture prepared for all genes being tested was as follows: $45 \mu \mathrm{l}$ of High Fidelity Elongase (Invitrogen) +10 $\mu \mathrm{M}$ each forward and reverse primers + dilution (1:10) of prepared cDNA. The gene-specific primer pairs were prepared by Invitrogen and 
PAGE purified: $f l k-1$ (286 bp) (Sandner et al., 1997) forward (5'CATGCTGTATCTCGAGGGC-3') and reverse (5' ${ }^{\prime}$-TTTGTCATCCCAGGGTAC- $3^{\prime}$ ) (40 cycles), flt-1 (238 bp) forward (5'-AGGATATTGTTCAGGGTCGAAGT-3') and reverse (5'-CAAGTGTAGAGTCCCTTG-3') (44 cycles), MAP-2 (422 bp) (Wang and Dow, 1998) forward (5'-GTCTGGTTTTACGGGTTGGCTGTC- ${ }^{\prime}$ ) and reverse (5'-GCCATGATCTTTCCCCTCTGGCTT-3') (28 cycles), and G3PDH forward (5'-TGAAGGTCGGTGTCAACGGATTTGGC- $\left.3^{\prime}\right)$ and reverse $\left(5^{\prime}\right.$ CATGTAGGCCATGAGGTCCACCAC-3') (21 cycles). Controls included omission of reverse transcriptase during cDNA synthesis. For each dose, at least three different rat brains were used for each experiment. The results shown were obtained from separate experiments repeated four times. Gels were scanned using Bioimaging Systems (Upland, CA) Gene Genius System, and the images were subsequently analyzed by the Gene Tools Analysis Software (Frederick, MD). After background correction, raw volumes representing densitometric values were obtained for each gel band. The raw volumes represented a semiquantitative guide to changes in mRNA expression.

Receptor antisense experiments. To distinguish the involvement of $f l t-1$ and $f l k-1$ in the observed effects of VEGF, antisense oligodeoxynucleotides (AS-ODN) were used. A set of modified 20-mer AS-ODNs targeted to $f l t-1$ and $f l k-1$ genes was synthesized commercially (Integrated DNA Technologies, Coralville, IA). To protect against nuclease degradation, all ODNs were end-capped by phosphorothioate modification at their $3^{\prime}$ terminal positions. The ODN sequences (Bernatchez et al., 1999) are as follows: $f l t-1,5^{\prime}$-GTCGCTCTTGGTGCTATA-3'; flk-1, 5' -CCTCCACTCTTTTCTCAG-3'. The following missense phosphorothioate sequence was used as a control: $f l k-1,5^{\prime}$-TGCTGGCATGTGCGTTGT-3'. Consistent effects on receptor translation are produced when ODNs are used at concentrations of $2-10 \mathrm{~mm}$ for $3 \mathrm{~d}$. AS-ODNs were added to the explant medium containing $0-100 \mathrm{ng} / \mathrm{ml}$ VEGF at the start of incubation; cultures were fixed with $4 \%$ paraformaldehyde and processed for immunocytochemistry on day 3.

Western blots. Homogenates of control and VEGF-antisense flk-1treated explants were prepared in RIPA buffer $(25 \mathrm{~mm}$ Tris, $150 \mathrm{~mm} \mathrm{KCl}$, 5 mм EDTA, 1\% Nonidet P-40, 0.5\% sodium deoxycholate, and $0.1 \%$ SDS, pH 7.4,) containing a protease inhibitor cocktail at the recommended concentration (Boehringer Mannheim, Ridgefield, CT). Twenty nanograms of a $43 \mathrm{kDa}$ flk-1-tagged fusion protein (Santa Cruz Biotechnology), used as a Western blotting control, and $250 \mu \mathrm{g}$ of total protein extracts were separated on 5\% Tris- $\mathrm{HCl}$ gels (Bio-Rad) and transferred electrophoretically to polyvinylidene difluoride membranes (Amersham Biosciences, Arlington Heights, IL). Membranes were probed using a flk-1 antibody (1:200; Santa Cruz Biotechnology). After incubation with the primary antibody, the membranes were washed three times with TBS-Tween (TBST) and then further incubated with horseradish peroxidase-conjugated secondary antibody (1:7500; Amersham Biosciences) for $1 \mathrm{hr}$. Subsequently, the membranes were washed three times with TBST and developed using the enhanced chemiluminescence plus detection system (Amersham Biosciences).

Signal transduction experiments. To study the role of MAPKK (ERK) in the explant cultures a MAP kinase (MEK1) inhibitor, PD98059 was introduced into VEGF-treated cultures and examined for neurite growth by TUJ1 or MAP-2 immunoexpression. PD98059 is a specific inhibitor of activated MEK1 in vitro and in vivo, which prevents the subsequent phosphorylation and activation of MAP kinase (ERK). PD98059 (50 $\mu \mathrm{M})$ (Promega, Madison, WI) was introduced into cultures that also received VEGF (50 or $100 \mathrm{ng} / \mathrm{ml}$ ) (the concentrations that produced the most consistent neuritic growth or maturation; see Results). Control cultures received serum-free medium alone, whereas another set of controls cultures were administered PD98059 (50 $\mu \mathrm{M})$ only. All cultures were incubated under standard conditions for $3 \mathrm{~d}$, followed by fixation in $4 \%$ paraformaldehyde and processed for immunofluorescence and confocal microscopy, as described previously. To investigate the possible involvement of the PI3-kinase pathway in VEGF signal transduction, wortmannin (50 mM) (Sigma), a potent PI3-K inhibitor, was added to cortical explants at the time of planting to serum-free medium containing 50 or $100 \mathrm{ng} / \mathrm{ml}$ VEGF. Control explants were incubated with wortmannin
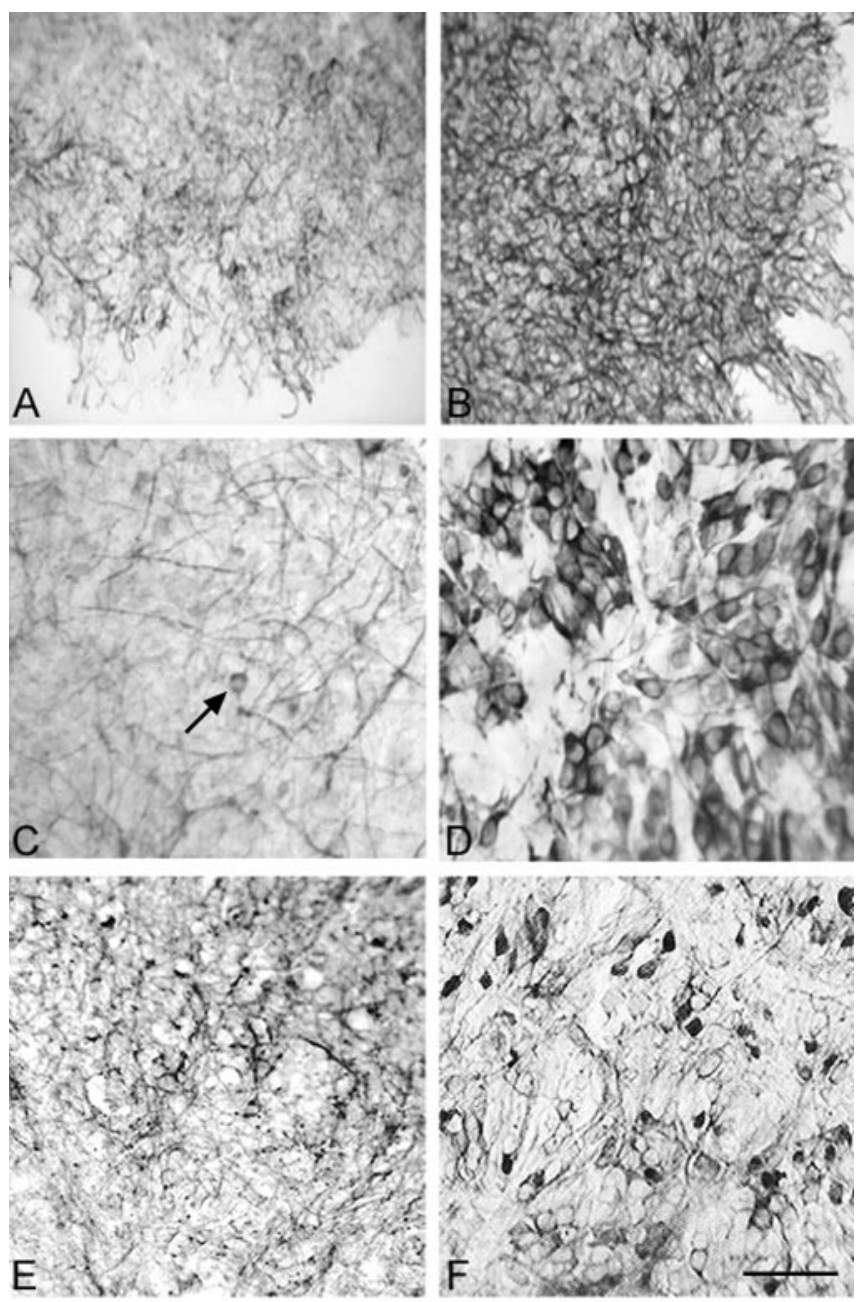

Figure 1. Neuronal protein immunoexpression in fetal organotypic explants. $A$, Survey micrograph of untreated explant has weak MAP-2 staining of cortical neurites. B, Sister explant treated with $50 \mathrm{ng} / \mathrm{mIVEGF}$ has intensely stained and thickened MAP- ${ }^{+}$neurites extending to the edge of the culture. $C$, Higher magnification shows that the effects on MAP- $2^{+}$neurite growth compared with control explants can be recognized after $10 \mathrm{ng} / \mathrm{ml} \mathrm{VEGF.} \mathrm{MAP-2}{ }^{+}$ processes are of moderate intensity, but neuronal soma staining, which was not observed in control explants, can be discerned (arrow). D, Application of $100 \mathrm{ng} / \mathrm{ml} \mathrm{VEGF} \mathrm{to} \mathrm{the} \mathrm{fetal} \mathrm{ex-}$ plants induces robust MAP-2 immunoexpression typical of mature cortical neurons. $E$, Untreated (control) fetal cortical explant has weak immunoexpression of NSE, a developmentally regulated neuronal glycolytic enzyme. $F$, Sister explant to $E$ treated with $100 \mathrm{ng} / \mathrm{ml}$ VEGF has a substantial increase in NSE immunoexpression and a much improved organizational appearance of the explant neuropil that was not seen in the controls or at low VEGF doses. NSE expression is representative of neuronal metabolic activity and the explant neurons had a variable staining intensity as occurs in situ.Scale bar: (in F) A, B, $140 \mu \mathrm{m} ; C, D, 100 \mu \mathrm{m} ; E, F, 120 \mu \mathrm{m}$.

alone. On day 3 of incubation, explants were fixed and processed for TUJ1 or MAP-2 immunofluorescence.

\section{Results}

\section{VEGF application causes upregulation of developmentally regulated neuronal proteins}

Organotypic fetal cortical explants cultured for $3 \mathrm{~d}$ in serum-free medium without VEGF (controls) typically survived reasonably well, but they exhibited only modest growth and sparse immunoexpression of MAP-2 within occasional neurites (Fig. 1A). MAP-2 is a developmentally regulated microtubular protein that is well recognized to be an important factor in neuronal growth and maturation (Matus, 1988; Tucker, 1990). During postnatal development, complete expression of MAP-2 is not reached until 


\section{MAP-2 IMMUNOREACTIVITY IN FETAL EXPLANTS}

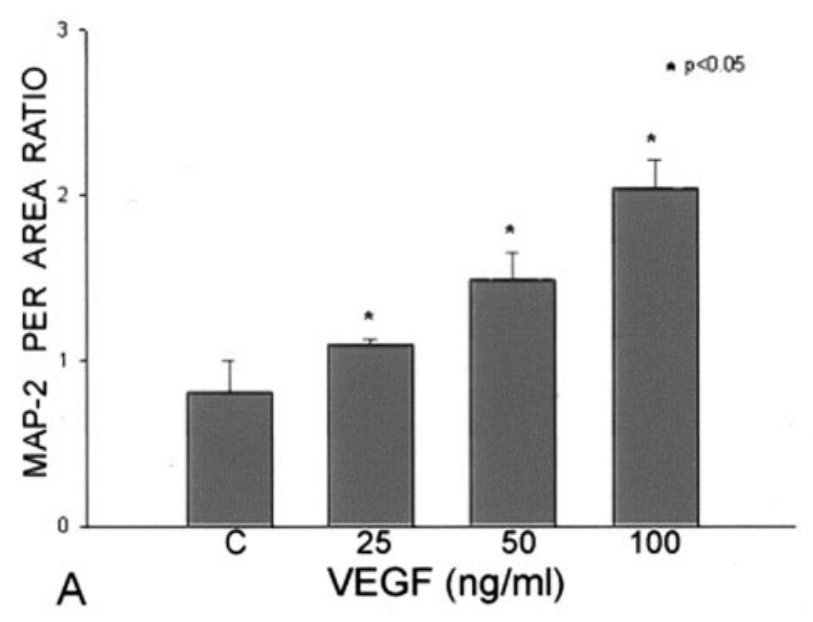

MAP-2 mRNA IN FETAL EXPLANTS

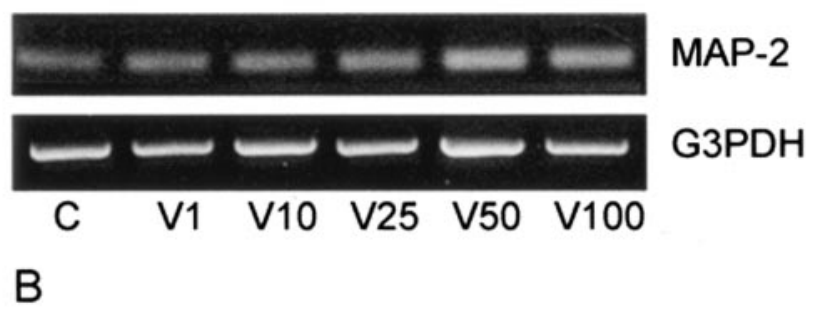

Figure 2. A, Semiquantitative analysis of MAP-2 immunostaining after VEGF application to the cortical explants using the Image-Pro and Metamorph systems (see Materials and Methods) depicts a significant dose-responsive increase ranging from $25 \%$ at $25 \mathrm{ng} / \mathrm{ml} \mathrm{VEGF} \mathrm{to}>100 \%$ at $100 \mathrm{ng} / \mathrm{ml} \mathrm{VEGF.} \mathrm{B,} \mathrm{Semiquantitative} \mathrm{RT-PCR} \mathrm{for} \mathrm{MAP-2} \mathrm{mRNA} \mathrm{also} \mathrm{shows} \mathrm{a} \mathrm{dose-responsive}$ increase after VEGF application compared with untreated explants, as measured by densitometric data from Gene Snap software (Cambridge, UK). The increase in MAP-2 message ranged from $30 \%$ at $1 \mathrm{ng} / \mathrm{ml}$ to $47 \%$ at $25 \mathrm{ng} / \mathrm{ml}$ to just over $100 \%$ at $50-100 \mathrm{ng} / \mathrm{ml} \mathrm{VEGF.} \mathrm{G3PDH,} \mathrm{Loading}$ control.

7-10 d (Matus, 1988; Rosenstein, 1995). In this organotypic explant model, MAP-2 remains at immature protein expression levels after $3 \mathrm{~d}$ in culture. In sister explants that received 50 or 100 ng of VEGF, substantial neurite growth was characterized by robust MAP-2 immunoexpression on a profuse carpet of thickened interconnecting and branching processes throughout the explants (Fig. 1B). At lower doses of VEGF (e.g., $10 \mathrm{ng}$ ) (Fig. 1C), an increase in MAP-2 expression within neuronal somata was initially recognized at higher magnification, whereas MAP-2 expression was never observed in neuronal somata of control explants. Cortical neurons within organotypic laminae in explants receiving 100 ng of VEGF had very strong MAP-2 cell-body expression (Fig. 1D), comparable with that of mature neurons in situ; such MAP-2 immunoexpression was not found in controls or even at lower VEGF doses. Quantitation of the MAP-2 expression of per-area ratio using Metamorph software showed that VEGF doses of 25-100 ng produced statistically significant increases in the microtubular protein (Fig. $2 \mathrm{~A}$ ). It should be noted that the profuse VEGF-induced growth within this explant model was not conducive to measuring neuronal individual sizes or branching of the neurites, although certain quantitative measures could be accomplished in primary neuronal cultures (see below). Semiquantitative RT-PCR showed a clear dose-responsive increase in MAP-2 gene product in the explants after VEGF appli- cation (Fig. $2 B$ ). The densitometry data derived from the Gene Snap software indicated that VEGF doses increased MAP-2 mRNA ranging between a $30 \%$ increase at $1 \mathrm{ng}$ to a $47 \%$ increase at $25 \mathrm{ng}$ to just over a $100 \%$ increase at $50-100 \mathrm{ng}$. Thus, VEGF application increased transcription of MAP-2, and its subsequent protein translation, to levels substantially above controls within the $3 \mathrm{~d}$ culture period.

We also examined the immunoexpression of NSE within the cortical explants using the same conditions. NSE is an enzyme specific to the neuronal glycolytic pathway that is developmentally regulated in situ and serves as a marker for neuronal maturation (Schmechel et al., 1978; Schmechel et al., 1980; Rosenstein, 1993). VEGF application at the higher concentrations (50-100 ng) produced immunostaining patterns comparable with those of MAP-2. Whereas control explants had sparse and fairly nonspecific NSE expression (Fig. 1E), VEGF-treated explants contained neurons that were strongly and discretely immunostained (Fig. $1 F)$. Although the VEGF-treated explants had demonstrably better (neuropil) organization, the degree of immunostaining intensity was somewhat variable within individual explant neurons as it occurs in situ in contrast to the MAP-2 data, in which all neurons exposed to $100 \mathrm{ng} / \mathrm{ml}$ VEGF were uniformly immunostained.

\section{VEGF application causes neuritic growth characterized by} increase in TUJ1 and flk-1 proteins

Organotypic cortical explants cultured for $3 \mathrm{~d}$ without VEGF and immunostained for the specific neuronal marker for TUJ1 contained more observable neurites (Fig. $3 A$ ) than did staining for MAP-2. This would be expected because TUJ1 is not postnatally regulated and is expressed in postmitotic neuronal somata. At the 10 ng VEGF dose there was a substantial increase in the number and size of TUJ1-expressing neurites (Fig. 3B). At the $100 \mathrm{ng}$ concentration, the neuronal profile in the explants had a much more mature appearance (Fig. $3 C$ ) that was comparable with the MAP-2 immunostained images (Fig. 2D). Robust TUJ1 immunostaining filled neurons with large cell bodies from which thick, intensely stained dendrites emanated. These results indicate that VEGF application, in separate experiments, markedly increases two different neuronal microtubular protein components in an almost entirely comparable manner in terms of neuritic and somal growth and maturation.

When the explants were double-immunostained for VEGF receptors, only flk-1 was coexpressed with TUJ1. Neuritic expression of $f l k-1$ was sparse in control explants (data not shown) but overlapped with most but not all TUJ1 ${ }^{+}$neurites at high VEGF concentrations such as $50 \mathrm{ng}$ (Fig. 3C, inset). Double immunostaining for $f l k-1$ and NeuN, a specific neuronal nuclear marker, depicted the dense neuritic outgrowth from a portion of a lamina containing cortical neurons after exposure to $100 \mathrm{ng} / \mathrm{ml} \mathrm{VEGF}$ (Fig. 3D). Probing for $f l k-1 \mathrm{mRNA}$ by RT-RCR showed a doseresponsive increase ranging from 30 to $120 \%$ after the $100 \mathrm{ng}$ VEGF dose (Fig. 3E). The presence of upregulated $f l k-1$ mRNA in these fetal brain explants after VEGF application is noteworthy because in adult brain it is practically undetectable by RT-RCR or Northern blot (Soker et al., 1998).

VEGF-induced neuritic growth is mediated by its flk-1 receptor and both the MAPK and PI3/Akt signaling pathways To determine the specificity of receptor-mediated neuritic outgrowth after VEGF application, we used AS-ODN technology to "knock down" the formation of the flk-1 and flt- 1 receptors.

When both 100 ng VEGF and a missense (control) ODN to 

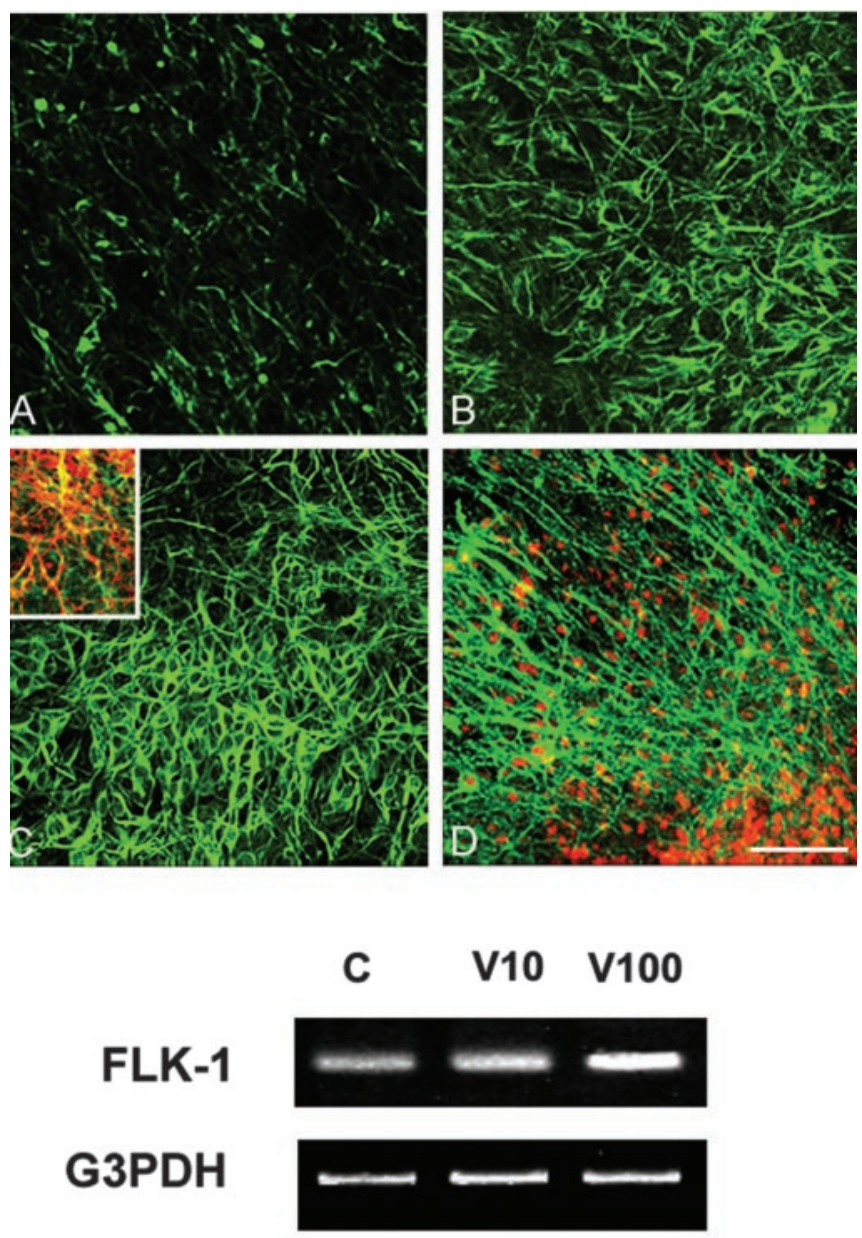

\section{$\mathbf{E}$}

Figure 3. VEGF-induced upregulation of TUJ1 and flk-1 immunoexpression in fetal cortical explants. $A$, Control cortical explant immunostained for TUJ1 shows moderate expression in fairly sparse neuritic processes. B, Sister explant treated with $10 \mathrm{ng} / \mathrm{ml} \mathrm{VEGF} \mathrm{has} \mathrm{stronger} \mathrm{TUJ1}$ immunoexpression in larger and thickened processes. C, After $100 \mathrm{ng} / \mathrm{ml} \mathrm{VEGF,} \mathrm{TUJ} 1$ expression in neuronal somata and processes is quite robust and comparable with that seen for MAP-2 expression at this dose (Fig. 1D). In untreated or low VEGF doses, strong TUJ1 or MAP-2 expression was not observed in neuronal somata. Inset, Double immunostaining for TUJ1 (green) and the VEGF flk-1 receptor (red) shows that portions of many, but not all, neuritic processes express this receptor (yellow). D, Double immunostaining for neuronal nuclear marker NeuN (red) and the flk-1 receptor (green) shows a profuse growth of thick neurites emerging from a cortical lamina in the organotypic culture after $100 \mathrm{ng} / \mathrm{ml}$ VEGF. Flk-1 immunoexpression in untreated cortical explants is sparse (data not shown), although its mRNA can be detected in E. Scale bar, $80 \mu \mathrm{m}$. E, Semiquantitative RT-PCR indicates a dose-responsive increase in flk-1 mRNA after VEGF application compared with untreated cultures ranging from $30 \%$ at $10 \mathrm{ng} / \mathrm{ml}$ to $120 \%$ at $100 \mathrm{ng} / \mathrm{ml} \mathrm{VEGF}$.

flk-1 were added to cortical explants, the neuronal appearance was similar to those incubated with $100 \mathrm{ng}$ VEGF alone (Fig. 4A). However, when the $f l k-1$ antisense ODN was added together with $100 \mathrm{ng}$ VEGF, there was a remarkable reduction in $\mathrm{TUJ}^{+}{ }^{+}$neurites that closely resembled control explants immunoreacted for TUJ1 (Fig. $4 B$ ). When the antisense ODN to $f l t-1$ was added with $100 \mathrm{ng}$ VEGF in the same paradigm, there was no change at all from the VEGF-only treatment. Western blotting experiments confirmed that the application of AS-ODN to flk-1 substantially reduced but did not eliminate the VEGF-induced increase in $f l k-1$ protein (Fig. 5). We have also found similar results in fetal ventral mesencephalic explants (our unpublished observations). These results indicate that the VEGF-induced neuritic growth is mediated specifically by the $f l k-1$ receptor.

To examine potential signaling pathways, we used two well known inhibitors in the explant model. The MAPK pathway has been shown in other systems to be inhibited by PD98059 (Alessi et al., 1995; Dudley et al., 1995). Compared with the robust TUJ $1{ }^{+}$neuritic growth after 50 ng VEGF (Fig. 4D) the addition of PD98059 nearly abolished the VEGF-induced outgrowth (Fig. $4 E)$. When the inhibitor was added to untreated sister explants there was no qualitative difference between these (Fig. $4 F$ ) and other control explants or the experimental ones that received 50 ng VEGF (Fig. 4E). To determine whether the PI3/Akt signaling pathway was operative we added the specific inhibitor wortmannin (Gerber et al., 1998) using the same paradigm. When wortmannin was added to explant cultures that also received $50 \mathrm{ng}$ VEGF, the robust TUJ1 ${ }^{+}$neuritic growth was nearly abolished (Fig. 4G). Control cultures that received wortmannin alone appeared entirely comparable with untreated controls as in the PD98059 experiments (data not shown).

\section{VEGF neurotrophic effects in primary neuronal cultures}

The organotypic explant model is useful to examine VEGF effects on developing cortical neuropil with the cellular components in a near-normal anatomical architecture. This raises the possibility that VEGF neurotrophic effects in this model could be of an indirect nature (see Discussion). We studied primary cortical neuronal cultures to eliminate potential interactions with other cell types and attempted to corroborate the observed neurotrophic effects observed in the explant model. It was evident morphologically that VEGF application had substantial effects on primary cortical neurons. Neurons cultured in serum-free medium without VEGF added had reasonable survival and neurite growth as seen in a survey micrograph (Fig. 6A). However, sister cultures that received VEGF application (10-100 ng) showed extensive neuritic outgrowth across the whole coverslip, as determined by TUJ1 immunoexpression (Fig. 6B). To exclude the possibility that we were just observing (and quantitating) more neurons, because VEGF has been described recently as a neuroprotective agent in hypoxic cultures (Jin et al., 2000a; Matsuzaki et al., 2001), we chose, on a random basis, relatively small, isolated clusters of neurons from each coverslip for morphological comparison and quantitation. A moderate-size cluster of neurons (six to eight) from cultures treated with $100 \mathrm{ng}$ VEGF had extensive neurite outgrowth and interconnections (Fig. 6D) compared with the untreated control (Fig. 6C). Similarly, an isolated neuronal doublet from a 50 ng VEGF-treated culture had a much more extensive neurite profile (Fig. $6 F$ ) than that from an untreated culture (Fig. 6E).

VEGF application to primary cortical neurons also induced strong upregulation of the $f l k-1$ receptor, whereas neurons in untreated cultures had modest $f l k-1$ immunoexpression that was associated only with the soma (data not shown). After a $50 \mathrm{ng}$ VEGF application, $f l k-1$ was expressed within the dendritic arborization of individual neurons (Fig. 6D). When AS-ODN to $f l k-1$ was added to $50 \mathrm{ng}$ VEGF-treated cultures, the extensive neuritic $f l k-1^{+}$outgrowth was mostly abrogated. These neurons were comparable in appearance for $f l k-1$ expression to untreated ones (Fig. $6 H$ ). These data strongly suggest that in both organotypic explants and primary culture, VEGF-induced neuritic growth is mediated directly through its $f l k-1$ receptor.

For quantitative purposes we chose to examine data parameters from TUJ1-immunostained cultures that could be analyzed in absolute terms such as neuronal size and number of neurites 

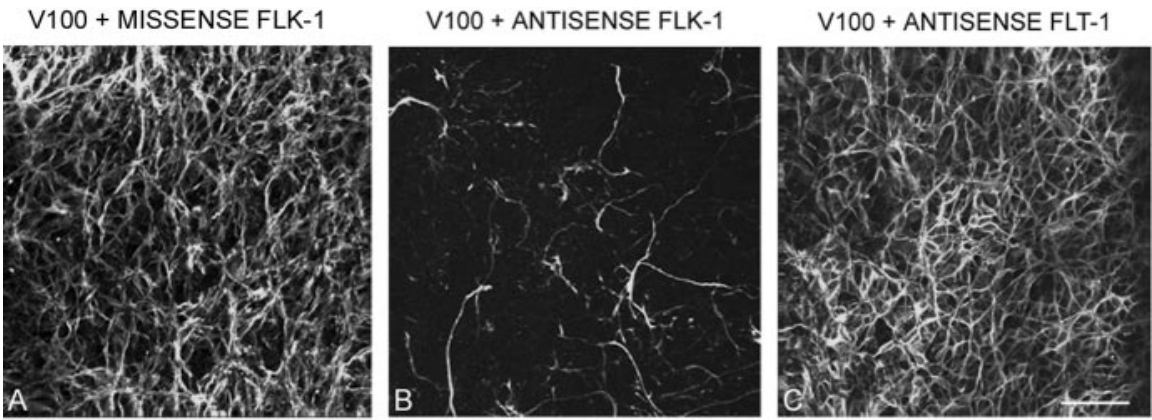

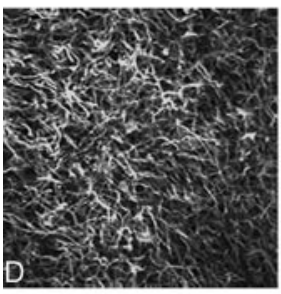

V50

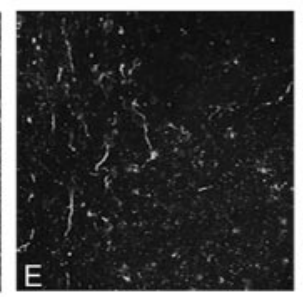

V50 + PD98059

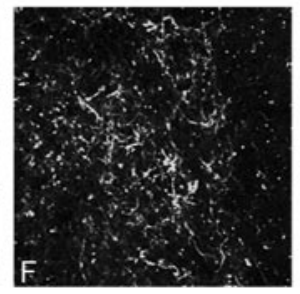

CONTROL + PD98059

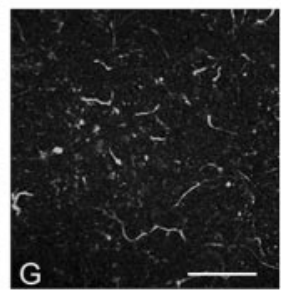

V50 + WORT
Figure 4. Receptor mediation and signaling pathways involved in VEGF-induced neuritic growth. A, Application of $100 \mathrm{ng} / \mathrm{ml}$ VEGF and a missense ODN sequence to flk-1 shows no change in VEGF-induced TUJ1 ${ }^{+}$neuritic growth. B, Application of $100 \mathrm{ng} / \mathrm{ml}$ VEGF and AS-ODN sequence to flk-1 shows a substantial decrease in TUJ1 ${ }^{+}$processes, reminiscent of an untreated explant. $C$, Application of $100 \mathrm{ng} / \mathrm{mIVEGF}$ and AS-ODN to the VEGF flt-1 receptor had no effects on neuritic growth. D, Application of $50 \mathrm{ng} / \mathrm{ml}$ VEGF to cortical explant shows strong MAP-2 ${ }^{+}$immunofluorescence. $E$, Addition of PD98059, a MAPK (MEK) signaling pathway inhibitor, nearly eliminates neuritic growth in a sister culture to D.F, When PD98059 was added to control explants there was little qualitative difference between these and explants that also received $50 \mathrm{ng} / \mathrm{mIVEGF}(E)$, ruling out the possibility that the inhibitor indiscriminately destroyed existing neurites. $G$, Addition of the P13-Akt signaling pathway inhibitor wortmannin to explants that also received $50 \mathrm{ng} / \mathrm{ml} \mathrm{VEGF} \mathrm{nearly} \mathrm{eliminated} \mathrm{neuritic} \mathrm{outgrowth} \mathrm{seen} \mathrm{with} \mathrm{VEGF} \mathrm{alone.} \mathrm{Application} \mathrm{of} \mathrm{wortmannin} \mathrm{to} \mathrm{untreated}$ cultures had no discernable effect (not illustrated). Scale bars: (in C) A-C, $100 \mu \mathrm{m}$; (in G) D-G, $125 \mu \mathrm{m}$.

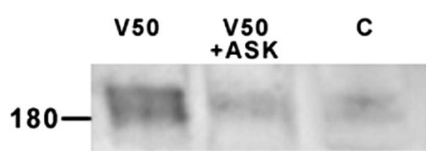

Figure 5. Antisense treatment of explants reduces flk-1 protein. Western blots show that the strong appearance of the flk- 1 doublet of protein bands at $\sim 180 \mathrm{kDa}$ after treatment with $50 \mathrm{ng} / \mathrm{ml} \mathrm{VEGF} \mathrm{(V50)} \mathrm{is} \mathrm{substantially} \mathrm{reduced} \mathrm{after} \mathrm{flk-1} \mathrm{AS-0DN} \mathrm{treatment} \mathrm{(V50} \mathrm{+} \mathrm{ASK)} \mathrm{and} \mathrm{is}$ comparable with the control (C).

directly emerging from a neuronal profile. In most instances neuritic growth was too extensive to measure branching points or absolute length accurately. At all VEGF doses (10-100 ng) significant trophic effects on primary neurons were found. After VEGF application, an increase in neuronal diameter ranged between 27 and $40 \%$ higher than control neurons (Fig. 7A). Quantitation of neurites emerging directly from the soma showed an increase over control ranging from $32 \%$ at $10 \mathrm{ng}$ to $60 \%$ at $100 \mathrm{ng}$ (Fig. $7 B)$.

\section{Discussion}

VEGF is nominally considered the most potent angiogenic factor in many biologic situations, including organogenesis, tissue repair, and tumor growth. In the brain, VEGF and its $f l k-1$ and $f l t-1$ receptors are normally quiescent, because the angiogenic component of the cerebrovasculature is practically nil. After a stroke or traumatic injury VEGF is intensely upregulated (Kovacs et al., 1996; Issa et al., 1999; Marti and Risau, 1999), and it has been suggested that it functions as a protective cytokine (Plate et al., 1999); its potential secretion by vascular cells, glia, or neurons could induce neovascularization so as to stabilize metabolic distress. Because of its strong expression in nonvascular cell types after trauma, studies have explored VEGF roles apart from vascularization, including astroglial proliferation and neuroprotection (see below). At this juncture it seems clear that VEGF plays pleiotrophic roles in the CNS that include cellular growth, proliferation, and protection (Oosthuyse, 2001; Jin et al., 2002).

In the present study we have used organotypic cortical explants as a model of neuropil development as well as primary cortical neurons to determine direct neurotrophic properties of VEGF on embryonic CNS neurons. Because of VEGF downregulation after birth and the lack of genetically modified animals (Fong et al., 1995; Shalaby et al., 1995; Carmeliet et al., 1996), hypotheses about the role(s) of VEGF in brain development have thus far been inferred from its observed upregulation after trauma (i.e., an increase in vascularization, stimulated largely by VEGF, would provide metabolic support for a growing neuronal population). A role for VEGF actions on CNS neuronal growth and development has not been detailed previously. A past study showed that VEGF application to ventral mesencephalic explants increased MAP-2 ${ }^{+}$neurites and promoted the survival of dopamine containing neurons (Silverman et al., 1999). However, neither receptor mediation nor signaling pathways were examined, and it was argued that other components in the explant cultures enhanced by VEGF could have contributed to neuritic growth (see below). Similarly, VEGF application to cultured adult dorsal root or autonomic ganglia (peripheral structures normally competent to regenerate) caused increased neuritic outgrowth and reduced apoptotic profiles compared with nontreated cultures (Sondell et al., 1999). Chemical inhibition of the flk-1 receptor and the MAPK signaling pathway attenuated the growth and survival effects; in addition, VEGF appeared to be mitogenic for Schwann cells. Therefore, VEGF appears to have nearly homologous actions on nonvascular elements both centrally and peripherally. In both systems VEGF induces neuritic growth and is also a potent mitogen for astroglia (Krum et al., 2002; Krum and Khaibullina, 2003) and Schwann cells (Sondell et al., 1999; Schratzberger et al., 2000).

The findings that VEGF application enhances neuronal growth within an organotypic explant model inherently present difficulties in accurately interpreting whether such effects are directly VEGF-receptor-mediated or indirectly mediated via factors produced by other neuropil cell types. Conceivably, after VEGF application the rapid increases in angiogenesis (Rosenstein et al., 1998) may initiate a cascade of signaling events or the production of endothelial-derived BDNF (Leventhal et al., 1999; Louissaint et al., 2002), which could then affect neuronal growth or migration. Moreover, astrocytes, when activated, can produce a substantial cadre of trophic factors, including VEGF (Eddleston and Mucke, 1993; Ijichi et al., 1995; Ment et al., 1997; Krum and Rosenstein, 1998), any number of which could interact with adjacent neurons. This scenario within the organotypic explant could be indicative of what might occur in situ, except for the absence of circulation and continual blood-brain barriercontrolled amino acid and glucose transfer from the blood. To 

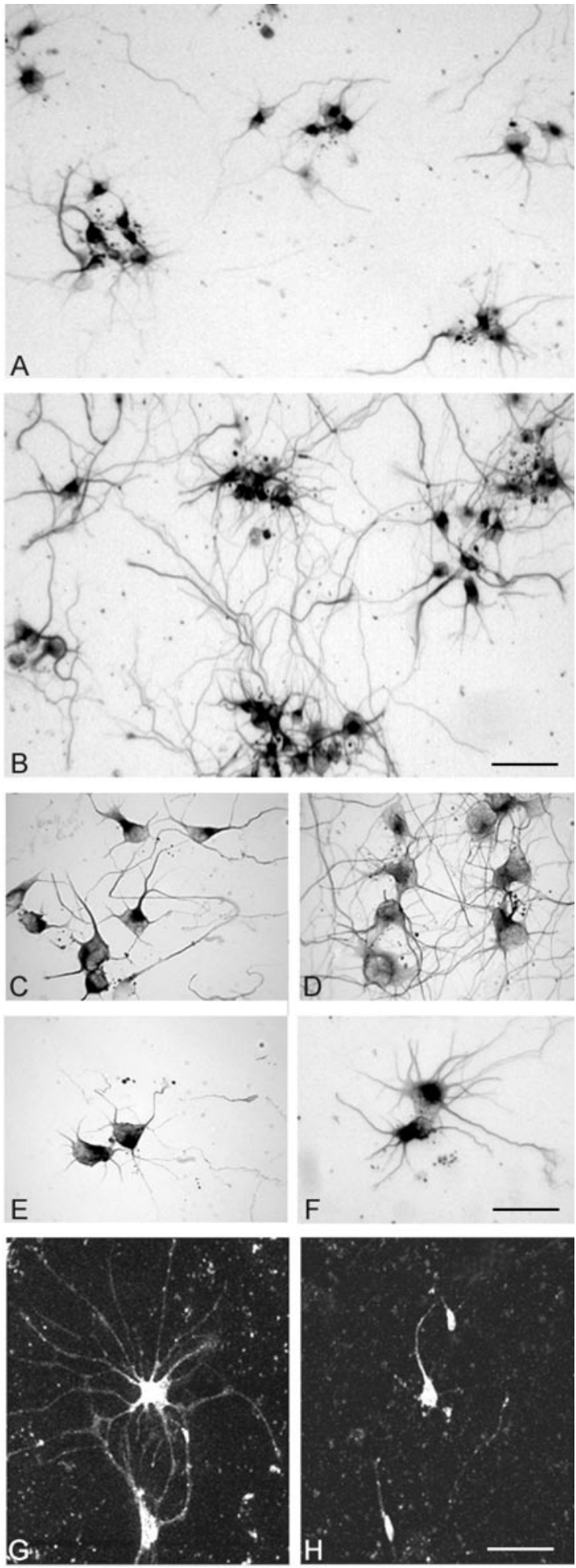

Figure 6. VEGF trophic effects on primary cortical neuronal cultures. $A$, Survey micrograph of untreated cultured primary cortical neurons grown in serum-free media immunostained for TUJ1 shows reasonable survival and growth. $B$, Survey micrograph of sister culture of cortical neurons after $100 \mathrm{ng} / \mathrm{ml} \mathrm{VEGF} \mathrm{shows} \mathrm{extensive} \mathrm{TUJ1}{ }^{+}$neuritic outgrowth extending between neuronal clusters. C, Clusters of TUJ1 ${ }^{+}$neurons in untreated culture. D, Neuronal cluster after $50 \mathrm{ng} / \mathrm{ml}$ VEGF shows longer and more branched interconnecting neuritic processes. E, Two isolated neurons in control culture. $F$, Pair of isolated neurons in a $10 \mathrm{ng} / \mathrm{ml} \mathrm{VEGF-treated} \mathrm{culture}$ show increased neuritic outgrowth from cell body. G, Isolated cortical neurons in serum-free primary culture after $50 \mathrm{ng} / \mathrm{ml} \mathrm{VEGF} \mathrm{immunostained} \mathrm{for} \mathrm{flk}-1$. H, Sister culture to $G$ shows lack of neuritic outgrowth from $\mathrm{flk}-1^{+}$neuronal somata after incubation with $50 \mathrm{ng} / \mathrm{ml} \mathrm{VEGF} \mathrm{with}$ AS-ODN to flk-1. Scale bars: (in $B) A, B, 100 \mu \mathrm{m}$; (in $F$ ) $C-F, 80 \mu \mathrm{m}$; (in $H) G, H, 100 \mu \mathrm{m}$.
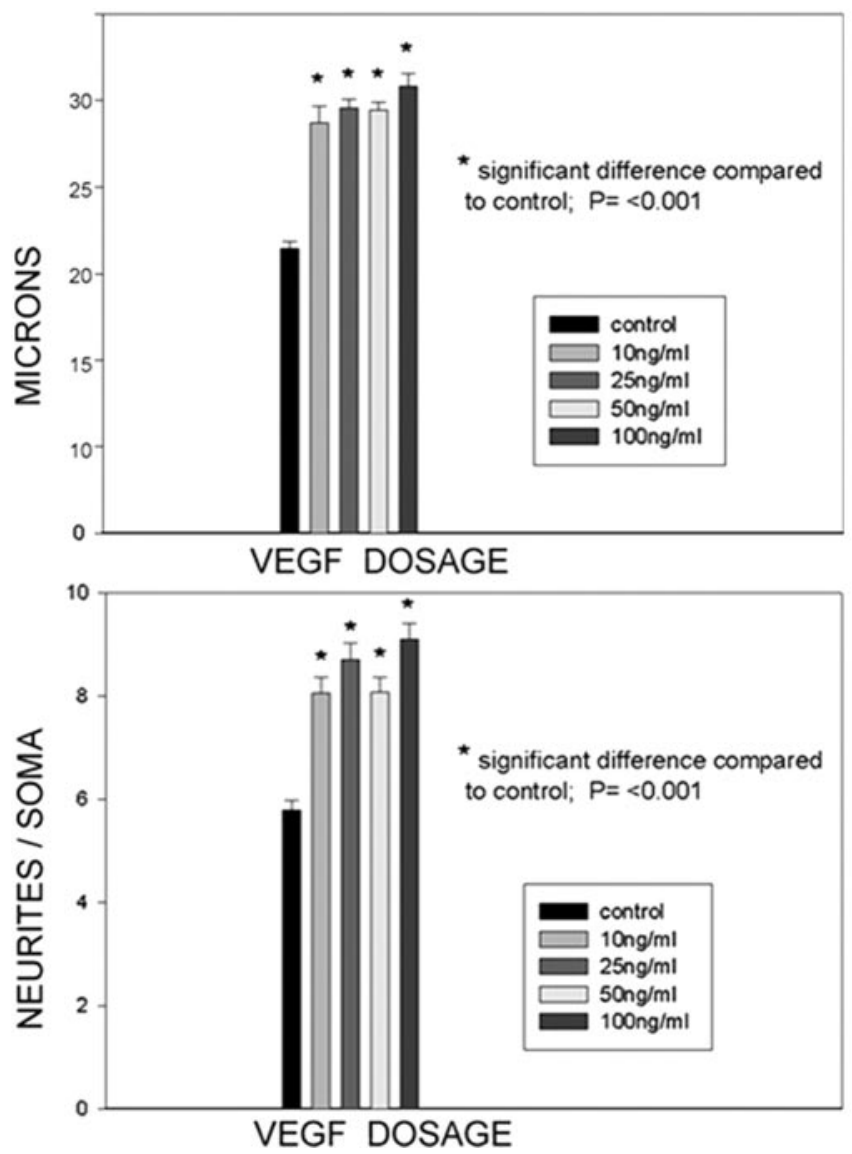

Figure 7. Quantitative assessment of VEGF-induced trophic effects on primary cortical neurons. Top, Absolute measurements of neuronal somata across the broadest diameter through the nucleus shows a significant increase in size at all VEGF doses ranging between $27 \%$ at 10 $\mathrm{ng} / \mathrm{ml}$ and $40 \%$ at $100 \mathrm{ng} / \mathrm{ml}$. Bottom, VEGF application produced a significant increase of neurites emerging from neuronal somata at all doses ranging up to $60 \%$ at $100 \mathrm{ng} / \mathrm{ml}$.

determine whether VEGF produced a direct neurotrophic effect, we also used primary cortical neuronal cultures, in which the potential contributions of stimulated endothelia and astrocytes were not an issue. The primary neuronal cultures allowed us to measure directly the substantial structural changes in VEGFtreated neurons with respect to somal size and neuritic outgrowth. In both the explant and primary culture models, addition of AS-ODN to the flk-1 receptor nearly abolished the neuritic outgrowth, whereas AS-ODN to the flt-1 receptor had no effect. In a previous study (Silverman et al., 1999), we applied neutralizing antibody to VEGF in ventral mesencephalic explants and found that it eliminated angiogenic effects but not neuritic outgrowth. It was suggested that VEGF receptors on vessels could be of lower affinity than those on neurons. Although specific receptor mediation of VEGF actions on neurons were demonstrated in the present study, it cannot be ruled out that other factors produced in the explant model (or potentially in an in situ situation) may have played an indirect or synergistic role in neuritic growth. An additional important avenue of investigation would be the examination of neuropilin, a coreceptor with $f l k-1$ for both VEGF and semaphorin; this association with a neuritic guidance system suggests that VEGF may be involved in the coordination of vascular and neuronal growth. It is interesting to note that VEGF application to primary cortical neurons produced comparable structural growth effects such as increased somal size and neurite 
outgrowth that GDNF induced on cultured primary dopamine neurons (Lin et al., 1993).

Recent studies have indicated that VEGF can play a neuroprotective role for cultured CNS neurons injured by either hypoxiaischemia, serum deprivation, or excitotoxicity (Jin et al., 2000a,b; Matsuzaki et al., 2001; Wick et al., 2002). In these models, VEGF application prevented neuronal apoptosis via activation of the PI3/Akt signaling cascade. However, an additional study showing VEGF protection of hippocampal neurons exposed to NMDA indicated that this pathway was not affected (Svensson et al., 2002). The protective effects demonstrated in these neuroprotection assays were mediated via the $f l k-1$ receptor. VEGF could target $f l k-1$ on neurons and promote signaling pathways that activate the Bcl-2 family for anti-apoptotic activity (Jin et al., 2000b; Matsuzaki et al., 2001). However, for challenged neurons that have functional glutamate receptors, the constitutive expression of $f l k-1$ or its coreceptor neuropilin-1 alone can mediate protection (Svensson et al., 2002). The present results show that both the PI3/Akt and the MAPK (MEK1) signaling pathways are involved in VEGF neurotrophic effects because the appropriate inhibitors nearly eliminated neuritic outgrowth. Our AS-ODN results indicate that the $f l k-1$ receptor mediates outgrowth, but AS-ODN to $f l k-1$ also blocked the neuroprotective effects of VEGF against glutamate toxicity (Matsuzaki et al., 2001), suggesting possible multiple roles for this receptor within the context of the PI3/Akt and MEK1 signaling pathways. The latter pathways may be operational in VEGF-mediated neuritic growth in the peripheral nervous system (Sondell et al., 1999), whereas the former pathway is well known for mediating cell survival. Whether there is greater neuronal survival in our normoxic VEGF-treated explants under serum-free conditions is currently under study. If VEGF application caused increased neuronal numbers within explants, it could account for the appearance of a more extensive carpet of neurites, but the primary culture data are strongly supportive of a direct VEGF-induced growth effect on individual neurons.

Although available data point to the neuroprotective actions of VEGF being mediated via the PI3/Akt or MEK1 pathways, mechanistically, on a cytoskeletal/cytoplasmic level, how VEGF achieves its neurotrophic effects remains to be elucidated. Microtubules are one of the most prominent cytoskeletal organelles and are involved in many important neuronal functions, including growth, structural stability, shape, plasticity, cAMP-dependent kinase phosphorylation, and maturation (Matus, 1988; Tucker, 1990; Hayes et al., 1995). VEGF application to CNS neuronal cultures upregulated both the expression and mRNA of MAP-2 and TUJ1, proteins that directly reflect the neuronal microtubule content, in a dose-responsive manner and quantitative differences in neurite content could be observed. It is an intriguing speculation that VEGF may affect the transcriptional regulation and translation of neuronal microtubular proteins and play a role in somatodendritic growth and maturation, although the upregulation of these proteins could be secondary to a different, as yet unknown, growth effect. Neuronal microtubules are well known to be rapidly downregulated (disassembled) by almost all forms of trauma, including hypoxia/ischemia (Kitagawa et al., 1989; Inuzuka et al., 1990; Folkerts et al., 1998). Because VEGF actions indicate an anti-apoptotic component (Jin et al., 2000; Larrivee and Karsan, 2000), it could have the potential to attenuate microtubule proteolytic dissolution that ultimately leads to cell death. Part of the neuroprotective activities of VEGF might include the modulation of both depolarization and activation of calcium-dependent proteolytic enzymes that serve to dissolve microtubular components. VEGF appears to upregulate certain developmentally regulated proteins, including NSE, a glycolytic enzyme unrelated to microtubular biology, that are downregulated in CNS injury. Whether VEGF can be shown to stabilize such proteins could have important ramifications for potential application in brain repair.

Although VEGF has long been recognized as a primary vascular proliferation factor, several studies have now shown that the cytokine has direct protective and trophic effects on neurons independent of a vascular component. Additional study of the subcellular effects of the VEGF molecule on different elements of the neuropil may reveal that it is involved in a number of seminal aspects of CNS development and repair.

\section{References}

Alessi DR, Cuenda A, Cohen P, Dudley DT, Saltiel AR (1995) PD 098059 is a specific inhibitor of the activation of mitogen-activated protein kinase kinase in vitro and in vivo. J Biol Chem 270:27489-27494.

Bernatchez PN, Soker S, Sirois MG (1999) Vascular endothelial growth factor effect on endothelial cell proliferation, migration, and plateletactivating factor synthesis is Flk-1-dependent. J Biol Chem 274:31047-31054.

Carmeliet P, Ferreira V, Breier G, Pollefeyt S, Kieckens L, Gertsenstein M, Fahrig M, Vandenhoeck A, Harpal K, Eberhardt C, Declercq C, Pawling J, Moons L, Collen D, Risau W, Nagy A (1996) Abnormal blood vessel development and lethality in embryos lacking a single VEGF allele. Nature 380:435-439.

Dudley DT, Pang L, Decker SJ, Bridges AJ, Saltiel AR (1995) A synthetic inhibitor of the mitogen-activated protein kinase cascade. Proc Natl Acad Sci USA 92:7686-7689.

Dvorak HF, Brown LF, Detmar M, Dvorak AM (1995) Vascular permeability factor/vascular endothelial growth factor, microvascular hyperpermeability, and angiogenesis. Am J Pathol 146:1029-1039.

Eddleston M, Mucke L (1993) Molecular profile of reactive astrocytes-implications for their role in neurologic disease. Neuroscience 54:15-36.

Ferrara N, Davis-Smyth T (1997) The biology of vascular endothelial growth factor. Endocr Rev 18:4-25.

Folkerts MM, Berman RF, Muizelaar JP, Rafols JA (1998) Disruption of MAP-2 immunostaining in rat hippocampus after traumatic brain injury. J Neurotrauma 15:349-363.

Fong GH, Rossant J, Gertsenstein M, Breitman ML (1995) Role of the Flt-1 receptor tyrosine kinase in regulating the assembly of vascular endothelium. Nature 376:66-70.

Gerber HP, McMurtrey A, Kowalski J, Yan M, Keyt BA, Dixit V, Ferrara N (1998) Vascular endothelial growth factor regulates endothelial cell survival through the phosphatidylinositol 3'-kinase/Akt signal transduction pathway: requirement for Flk-1/KDR activation. J Biol Chem 273:30336-30343.

Hayes RL, Yang K, Whitson JS, Postmantur R (1995) Cytoskeletal derangements following central nervous system injury: modulation by neurotrophic gene transfection. J Neurotrauma 12:933-941.

Hobson MI, Green CJ, Terenghi G (2000) VEGF enhances intraneural angiogenesis and improves nerve regeneration after axotomy. J Anat 197:591-605.

Ijichi A, Sakuma S, Tofilon PJ (1995) Hypoxia-induced vascular endothelial growth factor expression in normal rat astrocyte cultures. Glia 14:87-93.

Inuzuka T, Tamura A, Sato S, Kirino T, Yanagisawa K, Toyoshima I, Miyatake $\mathrm{T}$ (1990) Changes in the concentrations of cerebral proteins following occlusion of the middle cerebral artery in rats. Stroke 21:917-922.

Issa R, Krupinski J, Bujny T, Kumar S, Kaluza J, Kumar P (1999) Vascular endothelial growth factor and its receptor, KDR, in human brain tissue after ischemic stroke. Lab Invest 79:417-425.

Jin KL, Mao XO, Greenberg DA (2000a) Vascular endothelial growth factor: direct neuroprotective effect in in vitro ischemia. Proc Natl Acad Sci USA 97:10242-10247.

Jin KL, Mao XO, Greenberg DA (2000b) Vascular endothelial growth factor rescues HN33 neural cells from death induced by serum withdrawal. J Mol Neurosci 14:197-203.

Jin KL, Zhu Y, Sun Y, Mao XO, Xie L, Greenberg DA (2002) Vascular endothelial growth factor (VEGF) stimulates neurogenesis in vitro and in vivo. Proc Natl Acad Sci USA 99:11946-11950. 
Kitagawa K, Matsumoto M, Niinobe M, Mikoshiba K, Hata R, Ueda H, Handa N, Fukunaga R, Isaka Y, Kimura K, Kamada T (1989) Microtubule-associated protein 2 as a sensitive marker for cerebral ischemic damage-immunohistochemical investigation of dendritic damage. Neuroscience 31:401-411.

Kovacs Z, Ikezaki K, Samoto K, Inamura T, Fukui M (1996) VEGF and flt: expression time kinetics in rat brain infarct. Stroke 27:1865-1873.

Krum JM, Khaibullina A (2003) Inhibition of endogenous VEGF impedes revascularization and astroglial proliferation: roles for VEGF in brain repair. Exp Neurol 181:241-257.

Krum JM, Rosenstein JM (1998) VEGF mRNA and its receptor flt-1 are expressed in reactive astrocytes following neural grafting and tumor cell implantation in the adult CNS. Exp Neurol 154:57-65.

Krum JM, Mani N, Rosenstein JM (2002) Angiogenic and astroglial responses to vascular endothelial growth factor administration in adult rat brain. Neuroscience 110:589-604.

Larrivee B, Karsan A (2000) Signaling pathways induced by vascular endothelial growth factor. Int J Mol Med 5:447-456.

Leventhal C, Rafii S, Rafii D, Shahar A, Goldman SA (1999) Endothelial trophic support of neuronal production and recruitment from the adult mammalian subependyma. Mol Cell Neurosci 13:450-464.

Lin LF, Doherty DH, Lile JD, Bektesh S, Collins F (1993) GDNF: a glial cell line-derived neurotrophic factor for midbrain dopaminergic neurons. Science 260:1130-1132.

Louissaint Jr A, Rao S, Leventhal C, Goldman SA (2002) Coordinated interaction of neurogenesis and angiogenesis in the adult songbird brain. Neuron 34:945-960.

Marti HH, Risau W (1999) Angiogenesis in ischemic disease. Thromb Haemost 82:44-52.

Matsuzaki H, Tamatani M, Yamaguchi A, Namikawa K, Kiyama H, Vitek MP, Mitsuda N, Tohyama M (2001) Vascular endothelial growth factor rescues hippocampal neurons from glutamate-induced toxicity: signal transduction cascades. FASEB J 15:1218-1220.

Matus A (1988) Microtubule-associated proteins: their potential role in determining neuronal morphology. Annu Rev Neurosci 11:29-44.

Ment LR, Stewart WB, Fronc R, Seashore C, Mahooti S, Scaramuzzino D, Madri JA (1997) Vascular endothelial growth factor mediates reactive angiogenesis in the postnatal developing brain. Brain Res Dev Brain Res 100:52-61.

Oosthuyse B, Moons L, Storkebaum E, Beck H, Nuyens D, Brusselmans K, Van Dorpe J, Hellings P, Gorselink M, Heymans S, Theilmeier G, Dewerchin M, Laudenbach V, Vermylen P, Raat H, Acker T, Vleminckx V, Van Den Bosch L, Cashman N, Fujisawa H, et al. (2001) Deletion of the hypoxia-response element in the vascular endothelial growth factor promoter causes motor neuron degeneration. Nat Genet 28:131-138.

Plate KH, Beck H, Danner S, Allegrini PR, Wiessner C (1999) Cell type specific upregulation of vascular endothelial growth factor in an MCAocclusion model of cerebral infarct. J Neuropathol Exp Neurol $58: 654-666$.
Rosenstein JM (1993) Developmental expression of neuron-specific enolase immunoreactivity and cytochrome oxidase activity in neocortical transplants. Exp Neurol 124:208-218.

Rosenstein JM (1995) Diminished expression of microtubule-associated protein (MAP-2) and beta-tubulin as a putative marker for ischemic injury in neocortical transplants. Cell Transplant 4:83-91.

Rosenstein JM, Mani N, Silverman WF, Krum JM (1998) Patterns of brain angiogenesis after vascular endothelial growth factor administration in vitro and in vivo. Proc Natl Acad Sci USA 95:7086-7091.

Sandner P, Wolf K, Bergmaier U, Gess B, Kurtz A (1997) Hypoxia and cobalt stimulate vascular endothelial growth factor receptor gene expression in rats. Pflügers Arch Eur J Physiol 433:803-808.

Schmechel D, Marangos PJ, Zis AP, Brightman M, Goodwin FK (1978) Brain endolases as specific markers of neuronal and glial cells. Science 199:313-315.

Schmechel DE, Brightman MW, Marangos PJ (1980) Neurons switch from non-neuronal enolase to neuron-specific enolase during differentiation. Brain Res 190:195-214

Schratzberger P, Schratzberger G, Silver M, Curry C, Kearney M, Magner M Alroy J, Adelman LS, Weinberg DH, Ropper AH, Isner JM (2000) Favorable effect of VEGF gene transfer on ischemic peripheral neuropathy. Nat Med 6:405-413.

Shalaby F, Rossant J, Yamaguchi TP, Gertsenstein M, Wu XF, Breitman ML, Schuh AC (1995) Failure of blood-island formation and vasculogenesis in Flk-1-deficient mice. Nature 376:62-66.

Silverman WF, Krum JM, Mani N, Rosenstein JM (1999) Vascular, glial and neuronal effects of vascular endothelial growth factor in mesencephalic explant cultures. Neuroscience 90:1529-1541.

Soker S, Takashima S, Miao HQ, Neufeld G, Klagsbrun M (1998) Neuropilin-1 is expressed by endothelial and tumor cells as an isoformspecific receptor for vascular endothelial growth factor. Cell 92:735-745.

Sondell M, Lundborg G, Kanje M (1999) Vascular endothelial growth factor has neurotrophic activity and stimulates axonal outgrowth, enhancing cell survival and Schwann cell proliferation in the peripheral nervous system. J Neurosci 19:5731-5740.

Svensson B, Peters M, Konig HG, Poppe M, Levkau B, Rothermundt M, Arolt V, Kogel D, Prehn JH (2002) Vascular endothelial growth factor protects cultured rat hippocampal neurons against hypoxic injury via an antiexcitotoxic, caspase-independent mechanism. J Cereb Blood Flow Metab 22:1170-1175.

Tucker RP (1990) The roles of microtubule-associated proteins in brain morphogenesis: a review. Brain Res Brain Res Rev 15:101-120.

Wang W, Dow KE (1998) Quantitative analysis of mRNA expression of neuron-specific growth-associated genes in rat primary neurons by competitive RT-PCR. Brain Res Brain Res Protoc 2:199-208.

Wick A, Wick W, Waltenberger J, Weller M, Dichgans J, Schulz JB (2002) Neuroprotection by hypoxic preconditioning requires sequential activation of vascular endothelial growth factor receptor and Akt. J Neurosci 22:6401-6407. 